ness of the underlying derma. The effect was quite perfect.

Case 1.-A pril last $\mathrm{X}$ had a case of diphtheria, and it occurred to me to apply the acid pure. It was applied hourly to the patch on the tonsil, where it stuck from hour to hour, enveloped in the moisture of the parts, but gradually thinning the deposit and limiting its extent. It was the child of very poor parents, and after three days the mother reported such improvement that it was not again seen. The child recovered soon.

Case 2.-June 4. L. J., girl, æt. 8; well-marked membrane over one tonsil, with slight necrosis of deposit only; enlargement of lymphatics of neck ; some fever; no appetite. Powder of salicylic acid hourly, and $\mathrm{R}$ tinct. ferri chloridi $3 \mathrm{iv}$, hydrarg. bichloridi, gr. ij, m; gtt. iij in a teaspoonful of water every hour or two; acid every two hours at night. Continued nine days, with occasional intermissions of some hours, when the membrane would again appear, again to melt under the acid. No extension of the membrane while the acid was used. No complaint of the peppery stinging of the remedy. Towards the last, after disappearance of membrane, used also, $R$ bismuthi subnitratis $\bar{\jmath} \mathrm{ss}$, olei minthæ piperis, $m \mathrm{vj}$, m. Make a fine powder. Dust the throat every hour or two.

For a little space beyond the part affected by the membrane the salicylic acid whitens the mucous surface, but without inconvenience and without any invasion of the disease. The surface soon again recovers. The little patient was seen daily twice from June $4^{\text {th }}$ to $I I t h$, and to $I 3^{\text {th }}$ once daily, and last on the $\mathbf{r}^{\text {th }}$. No sequences.

Case 3.-Sept. 4. Mrs. H., æt. 33 ; considerable fever; enlarged glands of neck; weak; patch on one tonsil and near it.

B. Tinct. ferri chloridi 3 iv and hydrarg. bichloridi, gr. $i j$, me, gtt. iv in aqua $3 \mathrm{j}$ hourly or twohourly. Acid applied every hour or two; sometimes not so often. Complains of its stinging; membrane constantly recurs upon omission of acid. Finally applies remedy hourly with regularity, when the throat is no longer painful and the acid does not sting, and the deposit thins and disappears.

Mrs. H., a woman of intelligence, did not at first like the remedy, but seeing its benefit in the appearance and the lessened sensitiveness of the affected parts she became enthusiastic and thoroughly coöperated. She was visited twice daily for about nine days till Sept. I2th, when the tendency of the membrane to recur ceased. The bismuth mixture was then used with comfort.

A solvent of the membrane without irritation of the living structures seems to be found in the salicylic acid-much better than the subsulphate, whenever the membrane does not necrose and break down. The subsulphate hardens, in that way killing the acting membrane, rather than acting by solution. Creosote might act well but for its tendency to extend deeply.

The question of destructive action upon bacteria has not been discussed. It can in these in. stances be affirmed without discussion, and the treatment is considered from a different standpoint.

Hourly use of the acid, which is a very light powder, does not affect the youngest patient unfavorably by the amount swallowed or absorbed.

\section{FACT $V S$. FICTION TOUCHING YELLOW FEVER INOCULATION, WITH A RECORD OF RESULTS WELL AUTHENTICATED.}

\section{BY J. MCF. GASTON, M.D.,} FORMLRLY A RESIDENT OF BRAZIL.

The discussion of the claims of inoculation with the attenuated virus of yellow fever for the prevention of this disease or the mitigation of the access, should it be developed, resolves itself now into a consideration of facts. No further argument in regard to the principle upon which inoculation is based seems requisite for the proper understanding of the data which have accumulated illustrating its results. The only question likely to be made is respecting the correctness of the statistics and the trustworthiness of the reporter. But observing the rules which ordinarily determine the value of evidence it will be seen that quite a number of witnesses corroborate the statements of the original experiments. If the observations made and recorded by different individuals at different times and different localities, coincide in the points which substantiate the efficacy of yellow fever inoculation, no reasoning as to the theory of propagation, can invalidate the conclusions. True science deals with facts and not with theories, so that we must accept the evidence of those who report facts unless the credibility of the witnesses is impeached. As no interested motives can be attributed to the many Brazilian physicians who have recently investigated the process of inoculation introduced by Dr. Domingos Freire in Brazil, I will present the testimony as published in the Brazilian newspapers.

A fitting introduction to the report of observations from the city of Campinas, in the province of S. Paulo, is afforded by a letter addressed to the editor of the Gazeta de Noticias, of Rio de Janeiro, on May the I 2 th, I889, of which the following is a literal translation:

"I ask of you the kindness to insert in your valuable paper the accompanying letter from my distinguished colleague, Dr. Angelo Simōes; who has labored so diligently in Campinas for the active propagation of inoculation against the yellow fever. 
"By this letter it will be seen the same favorable results obtained here in the capital, as also in Nictheroy, Santos, Vassouras and Desengano, are being splendidly verified in the ill-fated Campinas; and I only regret that this was not done earlier for security against such evils and misfortunes. In due time I will publish the general statistics which place in evidence all the facts which militate in favor of the prophylactic measure, that from 1883 in four epidemics have clearly shown its value. In the meantime, while I recognize the support given by the press, a great part of the population and my colleagues; the ill-will of some, the ignorance and indifference of others have been obstacles to its general adoption, as is requisite for a complete effect of a preventative recourse which experience has fully sanctioned. Dr. Domingos Freire."

Following is the letter referred to above :

"Campinas, May 7th, r889.

"I received your letter of the 2 nd inst. and with it the culture liquid. I have vaccinated up to date 630 persons and $I$ think none remain to be vaccinated. I have gone through almost all the streets and meet only either those already vaccinated or individuals who have been attacked. One or another has refused to be vaccinated, but almost all have accepted it. I have proceeded with the greatest caution; I do not vaccinate those who have had the disease, nor those in the incubatory stage. I think, then, that the portion of the population not attacked by the disease have been vaccinated; and the new cases which are few, that are appearing in individuals who do not wish to avail themselves of this prophylactic, without counting one or another vaccinated, who has had the disease mildly.

"When the epidemic is ended I will prepare the statistics here for publication, for which I asi permission, presenting succinctly passing events without omitting anything.

"Withall I do not expect my labor to end with this. I intend to vaccinate, perhaps on a grand scale, those who return to the city with a view to protect them from the disease during the next year. -Dr. A. Simōes."

It is proper to state that my home in Brazil for a number of years was at the city of Campinas, and hence the facts connected with this outbreak of yellow fever among the people of this place, and the result of the use of inoculation for the arrest of the epidemic have a peculiar interest.

I have to assure my colleagues that what is herewith presented for their consideration is worthy of credit.

I cannot render a better service to the true history of inoculation than to present a few of the many notices which are recorded in the files of the newspapers of Campinas which are in my possession. These are sent by friends living in that section who have taken no part in the discussion of the merits of inoculation as a preventative of yellow fever.

The Correio de Campinas of April 25th, I889, contains the following statement:

"VAccination against THE Yei, IOW FeVER. -The distinguished physician Dr. Domingos Freire vaccinated yesterday in the Misericordia Hospital 34 persons, who went there to secure immunity from the terrible epidemic.

"Besides this many persons were vaccinated in their own houses.

"Dr. Domingo Friere was assisted by the noted practitioner, Dr. Angelo Simōes, who continues to aid the great scientific investigator.

"We continue to recommend to the people a resort to vaccination as the only preventative against the yellow fever.

"If all would avail themselves of that sure prophylactic, Campinas would be free from this great scourge in a few days."

We extract from another issue, May Ist, I889:

"Vaccination against yellow fever continues to be well received by the people.

"Yesterday, Dr. Ángelo Simōes, assisted by the pharmaceutist Soares da Camara, vaccinated 62 persons, completing the number of 510 who have been vaccinated.

"Up to the present time there is no notice of any of the persons vaccinated being attacked by the epidemic."

It is unnecessary to multiply this list of publications, but there are two points of importance which may be noted in the statements made:

"All the persons who have resorted to that preventative measure are in excellent condition, not one of them having been attacked by the epidemic.

"It is notable that on the day following the vaccination almost all the subjects perceive symptoms of fever, more or less marked; this fever, however, disappears without the use of any medicine.'

I may state that among the names of those vaccinated, many of my former acquaintances are recognized, who would be incapable of practicing any deception in this matter.

Those of my colleagues in this country who have known anything of my antecedents will not attribute to me a purpose to mislead them in regard to the facts developed by the large number of vaccinated having escaped unhurt, while others were dying around them. The great publicity given to the vaccination in the large hospitals and churches, with the records of the names and dates, affords an additional assurance of the trustworthiness of the statistics. It is not any longer requisite to send a commission to study this process from a scientific standpoint, but we have simply to accept or reject the testimony.

The result in the city of Campinas, in the 
province of S. Paulo, is shown by a telegram on May 3 rd, I889, as follows:

"Dr. Angelo Simōes, aided by the pharmaceutist Camara, has vaccinated more than six hundred persons by the process of Dr. Freire, having obtained excellent results. The epidemic is declining rapidly. The population is satisfied with the result of the vaccination."

This is taken from the Gazeta de Noticias, of Rio de Janeiro, and another telegram is published in the same paper from Campinas, May I Ith, as follows :

"Of the seven hundred vaccinated up to the present time only two have died of the yellow fever, yielding therefore an excellent result of this prophylactic measure."

For the information of the profession as to the effects of inoculation upon the subjects, the following report is translated from a newspaper of Rio de Janeiro, $O$ Paiz. It is by Dr. Urias A. da Silveira, author of "Brazilian Therapeutics," dated at Barra Mansa, August 29th, I 889, and addressed to Dr. Domingos Freire.

"Considering of utility, the two cases of vaccination against yellow fever done by you upon two persons of my family in your office at 68 Rosario street, as statistical elements, I come spontaneously to bring them to your attention. me:

'I present faithfully the facts as observed by

"Case $1 .-D . M ., 36$ years old, sanguine, nervous temperament, enjoying perfect health, vaccinated 3:30 P. M., July 25th, I 889 . General condition: At 6 P. M., hot flushes; at 8 P. M., the temperature in axilla 37.7 cent.; at midnight 38 . I cent.; at 6 A. M. on 26 th, general sweat without any medication and reduction of temperature to normal.

"Pains of the limbs, especially the lower extremities, frontal headache, vertigo, congestion of conjunctiva with running from eyes, slight yellow tint of conjunctiva perceptible to all. This followed by discomfort for twenty-four hours and tendency to drowsiness.

"Diminished appetite, urine scanty and highcolored, of which a sample is presented.

"At the outer and upper third of the left arm, where the injection of I gram of the culture liquid of the attenuated micrococco xanthogenico was made, there was observed six hours afterwards a red spot, of a congestive nature and slightly swollen, painful to the touch and pain increased by any movement. This spot continued for thirty hours, becoming then paler, less painful and less swollen. It gave place subsequently to a greenish-yellow discoloration, which disappeared after the seventh day.

"Case 2.-N. N., I4 years old, nervous lymphatic temperament and in perfect health.

"The same local and general phenomena described above were observed with little modifica-

tion, but more marked and accompanied with nausea and abundant flow of saliva. In both the signs of general disturbance preceded those of the 10calized lesion on the arms. The reunion of these signs in harmony and their development, demonstrates the results of abortive cases of yellow fever or that the symptoms correspond to it.

"Having these two facts of great significance, based on what I have learned from your elaborate work upon the vigorous method employed in the culture of the germs of yellow fever, and the attenuation of their violence, with due regard to what all bacteriologists practice, I am convinced that to you belongs the palm of victory for the discovery of the immediate cause of yellow fever and of the means of preventing it, with like benefit as the cow-pox in variola.

"A vaccination by the attenuated virus of yellow fever by the process of Freire is, in my humble opinion, the most efficacious hygienic measure of the present day, as it is the greatest factor employed for the extinction of yellow fever, already endemic in Brazil. Without it, fruitless and useless will be whatever means are employed to protect large cities. They may have the merit of diminishing their epidemic explosions but not to extinguish them entirely, as will occur if both should be employed together.

"Allow me, in conclusion, to express with frankness my opinion upon your process. It is that the vaccination of Freire is beginning to enter upon the scale of consummated facts, as one of the grand conquests of science over the greatest scourge of humanity.-Dr. Urias A. da Silveira."

In connection with these data relative to yellow fever inoculation from various sources, calculated to impress the medical profession with its efficacy, I will give a translation of the statistical report of the results in different portions of Brazil from I 883 to I 889 , recently published by Dr. Domingos Freire. While there have been announcements in the medical journals and in the newspapers of the United States, to the effect that Dr. George M. Sternberg has investigated this matter and pronounced an unfavorable decision in regard to the process of inoculation adopted by Dr. Freire and the results obtained by it, this matter is now submitted to the thinking man as a reality.

"Statistics of preventive inoculation against the yellow fever.

"The epidemic of yellow fever which was developed in Rio de Janeiro during $1888-89$, and was propagated to various places in the interior of Brazil, serves to demonstrate for the fourth time the prophylactic value of vaccinations by means of the attenuated microbe of this disease.

"It was between the months of December and March that the epidemic attained its greatest intensity; in the month of May of I 888 the first sporadic cases appeared, and the last in June, r889. We inoculated in this period 3,520 per- 
sons, of whom 988 were strangers and 2,532 Brazilians. Distributed thus: Rio de Janeiro, 2, I38; Campinas, 651 ; Vassouras, I99; Nitheroy, 163; Santos, I33; Desengano, IO2 ; Serraria, 80; Rezende, 54. The disease prevailed with great intensity at all these points.

"Among the 2,532 Brazilians are found I,680 that correspond very much to the 998 strangers, because in this number are embraced not only individuals coming from the interior and residents of this city for less than six years, but likewise children are included, which are proven to have a susceptibility similar to strangers themselves.

"The percentage of mortality was 0.79 among those who were vaccinated. In Santos, Rezende and Serraria the immunity of the vaccinated was complete. The percentage of each locality was the following: Rio, 0.98 ; Campinas, 0.46 ; Vassouras, 0.5 ; Nitheroy, 0.75 ; Santos, o; Deseugano, I.9; Serraria, o; Rezende, o.'

Let us see now the mortality from yellow fever among those not vaccinated. It was 4 , I I 5 , distributed as follows :

"Rio, 2,407 (including the sick of the Maritime Hospital of Santa Isabel); Campinas, 81 2 ; Vassouras, I 5; Nitheroy, I77 ; Santos, 650; Desengano, 22 ; Serraria, 21 ; and Rezende, II.

"Of the 4, I 5 there are about 2,800 strangers who died, being $1, \mathrm{r} 76$ in this city (Rio de Janeiro); 750 in the Maritime Hospital; 63 in Nitheroy; 500 more or less in Santos; 300 more or less in Campinas; 7 in Desengano ; 3 in Rezende; 3 in Vassouras.

"The deaths of Brazilians make up one-fourth, who for the most part have not been habituated to the virus, being in localities where the scourge has never before broken out.

"With a view to illustrate the efficacy of the inoculations, let us record the ratio adopted by Dr. Jemble in Senegal. He verified that among the strangers residing there from one to three years, 75 out of 100 are attacked with yellow fever and 66.6 out of roo die of it. Let us make application of these proportions to our vaccinated strangers or natives, that resided in the infected district from a few days to three years.

"There were inoculated in Rio de Janeiro I, I 83 persons under the conditions mentioned, and of these, the deaths should have been at least $59 \mathrm{I}$. Behold! only I 8 died; so that 573 lives were spared. In Campinas, a city never visited previously by yellow fever, the whole of the 65 I vaccinated may be considered as newcomers. Among these the deaths should have been 325 , whereas there were only 3 , and these were Brazilians, with complete immunity of $\mathrm{I}_{52}$ strangers.

"Likewise in Vassouras there should have died 5 , while there was only one victim. In Nitheroy there should have been 5 deaths among the I I strangers under the above conditions, yet there was but one fatal case among those vaccinated.
In Santos, in the list of 57 individuals under the same conditions, 28 should have succumbed, yet there was not a single case of death amongst our inoculated persons in this city. In Desengano the two unfavorable results were of strangers who had resided there six and eight years. In view of the fact that the disease prevailed in that place for the first time, all of the IO2 inoculated may be reckoned as susceptible in like manner as recently imported strangers. It might have been expected that at least $5 \mathrm{I}$ should have perished.

"In Serraria, making a similar calculation, 39 should have died, whereas the immunity was absolute. In Rezende the 54 inoculated should have furnished 27 fatal cases, as the disease had not appeared there previously, while in this city. there was complete immunity.

\section{"GENERAL SUMMARY.}

"From 1883 to 1889 we inoculated 10,474 persons with a mortality of 0.4 per hundred. From I 883 to I 884,4 I 8 inoculations; from I 884 to I 885 , 3,057 inoculations; from 1885 to $1886,3,473$ in1oculations; from 1888 to $1889,3,532$ inoculations. Total, 10,474 inoculations.

"The inoculations were interrupted during $\mathrm{I} 887$ on account of my visit to Europe and the United States, where I went to present the result of my efforts, which happily were well received.

"It is proper to note that with a view to avoid the criticisms of the ill-disposed, I have authenticated the results obtained this year; and I have the documents relating to the inoculations, all signed by eminent physicians, many of them sanitary authorities, by the members of municipal councils, by police officers and by many other distinguished and trustworthy persons. Would that this could have so happy an exit in the minds of those who are charged with the most precious of all interests--the health and the life of the people.-Dr. Domingos Freire.

"Rio, October 2, I 889."

With much labor and painstaking, these data have been collected and recorded, without any other object than the vindication of truth by facts. I am convinced that influences have been brought to bear upon the public mind in this country, which have prevented an impartial judgment in regard to the merits of this process of inoculation as a prophylactic against yellow fever. But with the report of such varied observations and statistics as are here presented, I am convinced that the members of the medical profession will not any longer shut their eyes to the overwhelming weight of testimony in favor of the protection afforded by this measure.

If our sanitary authorities will take steps to test yellow fever inoculation as it has been done in Brazil, all controversy will be ended by the realization of its protective agency in the arrest of yellow fever in our Southern cities. 